\title{
BMJ Open Earlier initiation of palliative care in the disease trajectory of people living with dementia: a scoping review protocol
}

\author{
Joni Gilissen (D) , ${ }^{1,2,3,4}$ Lauren Hunt, ${ }^{1,5}$ Lieve Van den Block, ${ }^{2,3}$ \\ Jenny van der Steen, ${ }^{6,7}$ Peggy Tahir, ${ }^{8}$ Christine Ritchie ${ }^{1,4,9}$
}

To cite: Gilissen J, Hunt L, Van den Block L, et al. Earlier initiation of palliative care in the disease trajectory of people living with dementia: a scoping review protocol. BMJ Open 2021;11:e044502. doi:10.1136/ bmjopen-2020-044502

- Prepublication history for this paper is available online. To view these files, please visit the journal online (http://dx.doi org/10.1136/bmjopen-2020044502).

Received 13 September 2020 Accepted 27 May 2021

Check for updates

(c) Author(s) (or their employer(s)) 2021. Re-use permitted under CC BY-NC. No commercial re-use. See rights and permissions. Published by BMJ.

For numbered affiliations see end of article.

Correspondence to

Dr Joni Gilissen;

joni.gilissen@gbhi.org

\section{ABSTRACT}

Introduction While the need for palliative care for people living with dementia has widely been recognised, they continue to be a disadvantaged group when it comes to timely initiation, and controversies remain regarding appropriate timing, or what elements constitute high quality palliative care early in the disease trajectory. To date, no literature review has summarised this debate or offered insights. The aim of this scoping review is to provide a general overview of research regarding palliative care in mild or moderate dementia, to identify existing controversies, and to examine what are key components of palliative care in dementia, specifically when initiated earlier in the disease trajectory.

Methods and analysis Consistent with recent guidelines and the Preferred Reporting Items for Systematic Reviews and Meta-Analyses Extension for Scoping Reviews, we carried out a search for academic literature in PubMed, CINAHL, EMBASE, Cochrane Library, PsycINFO, Web of Science; on 5 November 2019 and an updated search on 2 February 2021. We will include studies with different study designs published in English over the last decade that focused on palliative care in early stages of dementia. We will include models targeting at least one outcome domain of palliative care (physical, psychological, social or spiritual) and advance care planning, and will exclude hospice models with limited prognosis similar to the requirements in the USA. We will report study characteristics and quality. We aim to apply narrative synthesis techniques to develop a theoretical model of how, for whom and why palliative care can be relevant in early stages of dementia, and what are facilitators and barriers. We anticipate to also describe if and how the concept of (early) palliative care in dementia changed across time and studies.

Ethics and dissemination No ethical review required. Results will identify research gaps and lay out basic principles for conceptualising palliative care in early stages of dementia.

\section{BACKGROUND}

Dementia is characterised by a protracted course with progressive but insidious development of cognitive and physical disability. It has become an increasingly prevalent cause of death. ${ }^{12}$ By 2050, over $40 \%$ of adults aged 65 and older will die with or from dementia. ${ }^{13}$

\section{Strengths and limitations of this study}

The results of this review will establish a baseline understanding of the current state of affairs and controversies concerning palliative care early in the disease trajectory of people living with dementia-a timely and challenging theme among professionals.

- This protocol outlines a rigorous study design that includes the use of an established scoping review methodology, a search strategy informed by a multidisciplinary team, and a study selection and data extraction process that is carried out in tandem with all authors, whom are content experts.

- A potential limitation of the review is the lack of definition of 'early palliative care' and 'palliative care early in the disease trajectory of dementia', which points to the necessity of this scoping review.

- Studies will not be excluded based on their quality assessment as this review aims to provide a snapshot of the current landscape and scientific debates by being inclusive of all types of information available in academic literature.

Although there is widespread recognition that palliative care has much to offer people living with dementia and their loved ones ${ }^{45}$ from the time of diagnosis across the disease course ${ }^{67}$ people with dementia are consistently shown to be a disadvantaged group when it comes to timely and high-quality palliative care.

Palliative care focuses on improving the quality of life of individuals and their families facing problems associated with serious illness through the prevention and relief of suffering by means of early identification and impeccable assessment and treatment of pain and other problems, physical, psychosocial and spiritual. ${ }^{8-10}$ However, despite dementia being associated with decreased life expectancy, many do not perceive it to be lifelimiting or terminal at time of diagnosis or earlier in the course of disease. ${ }^{2}$ Lack of access to palliative care results in a large amount of unmet needs, a high burden of pain, other sources of distress, and frequent burdensome 
hospitalisations and invasive procedures-that neither improve the length nor the quality of life. ${ }^{1511}$ Time trend analysis of population-based data in Belgium shows that over the years, the timing of initiating specialist palliative care services (such as palliative care home team, palliative care unit, palliative care team in nursing home or mobile palliative care support hospital-based team) in dementia continues to occur very close to death. ${ }^{12}$ This is consistent with other international literature showing that palliative care is generally initiated late for all conditions; especially in dementia, at a median (P25-75) up to only 14 days (7-30) before death. ${ }^{13}{ }^{14}$ In the USA, although there has been a large increase in access to hospice for people with dementia to the point that almost half of all hospice recipients annually have a primary or comorbid diagnosis of dementia, ${ }^{5} 1516$ people with dementia still only have a median length of stay in hospice of only 17 days, and many exceed the 6-month time limit, with live discharge.${ }^{17}$ In addition, almost half of national dementia plans around the world lack any reference to end-of-life care or palliative care. ${ }^{18}$

There has been much debate about palliative care in the early stages of the dementia trajectory, ${ }^{19-21}$ with a variety of opinions between healthcare professionals. ${ }^{19} 22$ There are controversies regarding the timing and appropriateness of adopting a palliative care approach, and what it should constitute. ${ }^{19-21}$

Professionals across Europe and internationally have different opinions regarding the time point to when to consider a person with dementia in need of palliative care. ${ }^{192-24}$ The spectrum of experience in dementia is argued to be both vast and profound-changes may be relatively easy to manage or extremely complex, ${ }^{2}$ which can be compounded by an unpredictable disease course and the potential for people to have a prolonged experience of its most advanced and debilitating state. ${ }^{25} 26$ Despite the call for research into palliative care across different stages of dementia, ${ }^{27}$ it has been argued prognostication for palliative care initiation depending on stages of dementia might be challenging ${ }^{8}$ - people with dementia in earlier stages may be in the terminal phase; patients may survive to the last phase of dementia with severe physical and cognitive impairment ${ }^{1}$ but may also die earlier from comorbid conditions for example. While some argue a more advanced stage of dementia is the best time to start palliative care, ${ }^{19}$ others argue that waiting until the advanced stage of the illness might reduce opportunities for improving quality of life for the patient and caregiver earlier in the disease course, ${ }^{28}$ including the option to still have meaningful conversations. Anticipating the progression of the disease by initiating advance care planning when the patient can still be actively involved and when patient preferences, values, needs and beliefs can be elicited, argues for earlier palliative care involvement ${ }^{8}$ or social and spiritual support. ${ }^{8}$ Then there are those arguing palliative care initiation should not depend on prognosis or age, rather on care needs. ${ }^{29} 30$
Appropriateness of palliative care early on is sometimes questioned. ${ }^{23}$ A recent study for example reports that specialist palliative care services in dementia were not initiated because of physicians' consideration of palliative care 'as not being meaningful' in dementia. ${ }^{31}$ Earlier initiation of palliative care is sometimes perceived to add to distress, or perceived loss of hope for the person with the condition and their family members. ${ }^{20}{ }^{32}$ However, there is growing interdisciplinary consensus that the concept of a relatively short period of decline towards death is uncommon in persons with dementia and an increasing number of older people with dementia will experience prolonged periods, possibly many years (eg, young-onset dementia ${ }^{33}$ ), of profound disability where any exacerbation of their condition could potentially be fatal and where palliative care is extremely valuable. ${ }^{34}$

It is still unclear how to shape palliative care in dementia in practice and what effective care would look like at different points in the dementia trajectory, especially early on. ${ }^{23}$ We currently lack insight into what types of services should/can be offered at an earlier stage, which term is preferred (notion of 'supportive care' has been proposed ${ }^{35}$ ), and how this all relates to what are key components palliative care early in the disease course, ${ }^{23}$ beyond proactive (care) planning. ${ }^{8} 192022$ Nor has there been an exploration of how these controversies vary between countries and healthcare systems, which may have different approaches to dementia palliative care. ${ }^{36-38}$

Hence, while the general zeitgeist seems to call on timely and early initiation of palliative care in dementia, ${ }^{839}$ there is no literature review that to date has summarised the scientific debate or offered insights on what combination of elements constitutes high-quality timely palliative care. While multiple reviews on dementia and palliative care have been published, ${ }^{27} 4041$ these have focused on palliative care at the end-of-life, terminal or advanced stages of the disease $\mathrm{e}^{4243}$ or were limited to specific settings (eg, long-term care facilities or at home ${ }^{44}{ }^{44}$ ). Other reviews specifically aimed to evaluate effects of palliative care, ${ }^{45}$ barriers and facilitators for its optimal implementation, or how people experience palliative care by various perspectives. $^{44} 4647$

\section{General aim and research questions}

We aim to conduct a scoping review of the literature in order to systematically map the research done in palliative care early in the disease trajectory of people living with mild and moderate dementia, as well as to identify any existing gaps in knowledge, current scientific debates and conceptualisations. Early palliative care in this study is defined as 'in the early stages of the disease trajectory of dementia', starting from diagnosis. Hence, not focused on people living with advanced, end-stage, terminal stages of dementia. Palliative care in this review is conceptualised as defined by the $\mathrm{WHO},{ }^{5}$ that is related to one, more or all of its domains (physical, social, psychological, spiritual) and can be provided by any type of generalist or specialist (palliative care) healthcare provider. Generalist 
palliative care most often being provided by generalist healthcare professionals and/or providers not specialised in palliative care. Specialist palliative care intended to be provided by professionals (and the services they compose) that work solely in the field of palliative care and whose main activity is devoted to dealing with complex problems requiring specialist palliative care skills and competencies. $^{44} 48$

Guiding research questions-among others that we may derive from the review data-include:

1. How is 'early', 'timely', 'earlier' palliative care defined, in relation to dementia?

1. What are current debates in the scientific literature? What are pros and cons?

2. What are key components of palliative care early in the disease trajectory of people living with dementia?

3. What are differences between general and specialist palliative care, when provided early in the dementia disease trajectory?

4. Are there differences in the provision of palliative care early in the dementia disease trajectory, between healthcare settings?

5. What are important events that have shaped/ changed the definition of palliative care and to what extent?

2. What are palliative care needs in people living with dementia early in their disease trajectory? And how are these defined and identified?

3. What are barriers and/or facilitators for initiating palliative care early in the disease trajectory?
4. What are potential outcomes of integration of palliative care early in the disease trajectory, for all those involved in the provision or receipt of dementia care (including their family and professional caregivers)?

\section{METHODS}

\section{Scoping review}

Scoping reviews apply systematic principles to reviewing the literature for the purposes of examining the extent, range and scope of research activity, addressing a broad review question, and including all available research, irrespective of study design. ${ }^{49}{ }^{50}$ We have decided to conduct a scoping review as opposed to a systematic review given the lack of clear definition of what constitutes 'early palliative care' or 'palliative care earlier in the disease trajectory'. We will perform a scoping review consistent with the methodological framework proposed by Arksey and O'Malley, refined by Levac et al. ${ }^{49}$ It will integrate qualitative and quantitative evidence through narrative juxtaposition (discussing diverse forms of evidence side by side) ${ }^{51}$ This study protocol has been constructed following the Preferred Reporting Items for Systematic Reviews and Meta-Analyses (PRISMA) Extension for Scoping Reviews guidelines. $^{52}$

\section{Databases and search strategy}

We will search the following key international databases: MEDLINE (PubMed), The Cochrane Library, CINAHL, PsycINFO, EMBASE and Web of Science (table 1). No grey literature and texts that are not peer-reviewed are

Table 1 Search string

\section{PubMed}

(dementia OR "Dementia"[mesh]) AND (((early OR earlierOR timely) AND "Palliative Care"[Mesh]) OR "early palliativecare" OR "earlier palliative care" OR "Hospice Care"[Mesh]OR "hospice care"[tiab] OR "Terminal Care"[Mesh] OR"terminal care"[tiab]) LIMITS: human, 2009-2019

\section{EMBASE}

('dementia'/exp OR dementia OR 'alzheimer disease'/ exp OR 'alzheimer disease') AND (('palliative care'/exp OR 'palliative care') AND (early OR earlier OR timely) OR 'early palliative care' OR 'earlier palliative care' OR 'timely palliative care' OR 'hospice care'/exp OR 'hospice care' OR 'terminal care'/exp OR 'terminal care') LIMITS: human, 2009-2019

\section{PsycINFO}

(dementia or alzheimer or alzheimer's) AND ("early palliative care" OR "earlier palliative care" OR "timely palliative care" OR "hospice care" OR "terminal care") LIMITS: Peer review, 2009-2019

\section{Web of Science}

TOPIC: (dementia OR alzheimer OR “alzheimer's") AND TOPIC:

("early palliative care" OR "earlier palliative care" OR "timely palliative care" OR "hospice care" OR "terminal care")

\section{CINAHL}

(dementia or alzheimer or alzheimer's) AND ("early palliative care" OR "earlier palliative care" OR "timely palliative care" OR "hospice care" OR "terminal care")

LIMITS: Academic journals, 2009-2019

\section{The Cochrane Library}

Dementia "early palliative care"; Dementia "earlier palliative care"; Dementia "timely palliative care"; Dementia "hospice care"; Dementia "terminal care"; Alzheimer "early palliative care"; Alzheimer "earlier palliative care"; Alzheimer "timely palliative care"; Alzheimer "hospice care"; Alzheimer "terminal care"; Alzheimer's "early palliative care"; Alzheimer's "earlier palliative care"; Alzheimer's "timely palliative care"; Alzheimer's "hospice care"; Alzheimer's "terminal care" 
included because we are mainly highlighting scientific debates. The full search strategy is presented in table 1 and was built by an experienced medical librarian (PT), taking into account two validated palliative care literature searches. ${ }^{53}{ }^{54}$ In a final search phase, relevant literature reviews and key reports will be handsearched to identify articles missed by the search strategy. If-before submission of final results- the search was conducted more than a year ago, we will update our search. We included studies reported in English, with a publication date between January 1, 2009 and the date of search. One author (PT) downloaded all retrieved records, compiled them by using reference manager (Zotero), and removed duplicate records. A first search was conducted on 5 November 2019; an updated search was performed on 5 February 2021.

\section{Inclusion and exclusion criteria}

Criteria are outlined in table 2. We particularly include peer-reviewed research studies with different study designs, and reviews. Studies can include different palliative care domains (physical, psychological, social, spiritual) and should relate to the nature of the care provided, not the degree of training and/or experience of those providing care. We consider papers to be eligible when palliative care is provided inter-professionally, or by any type of healthcare provider (general practitioner, geriatrician, pharmacist, team, etc), and can include both generalist and specialist palliative care (providers). All settings are eligible, except for studies focusing on hospice(s) in USA and other countries with similar regulations related to a prognosis of 6 months of life or less. Papers can report on interventions (including those aimed at advance care planning, shared decision making or palliative sedation with the aim to improve quality of life), services delivery models, programmes, approaches to care or support, specific activities, tools, questionnaires. Papers with an exclusive focus on 'advanced', 'end-stage', 'late-stage' or 'terminal phases/stage of' or 'terminal' dementia; 'people with dementia drawing close to death' (having FAST (Functional Assessment Staging) score of 7c and up-routine eligibility criteria for hospice in the US; a CDR (Clinical Dementia Rating) score of more or equal to 2; or an MMSE (Mini-Mental State Examinition) score of 11 or less),${ }^{55-57}$ are excluded on screening to ensure focus is on early stages of disease. We include all types of dementia (examples in table 2).

\section{Study screening and selection}

Studies are selected in two phases, first by title-abstract screening, then by full-text screening. In the first phase, one author (JG) will screen titles and abstracts and exclude records not fulfilling the inclusion criteria. A list of studies labelled to be included for full-text screening, is then sent to a second author (LH), as well as the list with studies of which the first author is doubting inclusion. The list of excluded studies is sent to the other authors $(\mathrm{LVdB}, \mathrm{JvdS}$ and CR) who will independently screen a random sample
( $10 \%$ in total). In a second phase, we will obtain the fulltext documents and JG will check for eligibility; which will be discussed with LH. In cases of divergences, a consensus will be reached by discussion with the other researchers ( $\mathrm{LVdB}, \mathrm{JvdS})$, and if necessary, by consultation with an arbiter (CR). Study selection and screening is performed online via Rayyan QCRI. We plan to include a PRISMA flow diagram in the full review report to document the screening process, including the number of studies that were excluded and primary reason for their exclusion. ${ }^{58}$

\section{Quality assessment and risk of bias}

We will conduct a critical appraisal of included sources of evidence and describe the methods used in each study in a table summarising the characteristics of included studies. Two authors will independently assess the quality of the studies, using the Joanna Briggs Institute Critical Appraisal Tools for use in systematic reviews. Quality assessment will be reported but studies are not excluded based on their quality score.

\section{Data extraction and management}

Included studies will be uploaded in NVivo software and data will be extracted and charted by the first author (JG) ${ }^{49}$ We will abstract data on article characteristics and results of each study along with quotes (cf. 'excerpts'), themes and concepts pertinent to our research questions from both results and discussion sections of the included studies. Evidence from systematic reviews and primary studies will be checked and any overlapping data will be reported to avoid over-reporting.

\section{Narrative analysis and synthesis}

We aim to apply a narrative synthesis, guided by several techniques. ${ }^{5758}$

Included studies will organised into groups/clusters, a common technique in narrative synthesis ${ }^{5758}$ and a useful way of aiding the process of description and analysis and looking for patterns within and across these groups. Groups are refined as the synthesis develops.

We will perform a thematic content analysis of the included studies. We will code relevant excerpts, words and text into recurring and prominent themes, via deductive coding, guided by our research questions, ${ }^{59}$ and inductive coding. ${ }^{60}$ Identified themes will be refined through an iterative process by JG, in close collaboration with a second author. Final themes will be reviewed and discussed by all authors to reach consensus about categories that are reported in final synthesis. Because this is an iterative process, we aim to describe the main revisions with a rationale, to increase transparency of reporting. This is the most common method adopted within scoping reviews to produce a relatively rudimentary synthesis of findings across the included studies. Separate analysis of subgroups or subsets is not planned a priori. If possible, we will report disadvantaged and minority groups separately, if sufficient data are available. We will also explore differences in different types of dementia (eg, Alzheimer's, 
Table 2 Inclusion and exclusion criteria study selection

\begin{tabular}{lll}
\hline & Inclusion criteria & Exclusion criteria \\
\hline Publication date & All connected by 'AND' & All connected by 'OR' \\
\hline & $\begin{array}{l}\text { Between (1 January) 2009 until date of } \\
\text { search }\end{array}$ &
\end{tabular}

Type of publication

- English

- Peer-reviewed

Duplicate, congress reports or abstract, book or book chapter, opinion piece, editorial or discussion article, letter to the editor, individual case report, evaluation of local programme/audit, study protocol, report of consensus meeting(s), questionnaire or training session or $\mathrm{PhD}$ thesis.

Population

\section{Subject/intervention}

Humans

Adults

- All types of dementia (all types*, defined and identified in any way) 'OR' adult family or professional (health, social) caregivers 'OR' volunteers providing/ facilitating support

- Early stages of the disease trajectory
- Animals

Children

- Adolescents (<18 years old)

- Papers focusing solely on people living with 'advanced', 'end-stage' or 'late-stage' or 'terminal phases/stage of' or 'terminal' dementia; 'people with dementia drawing close to death' (having FAST score of 7c and up; a CDR score of more than 2 (include score of 2); an MMSE score of 11 or less).

Studies focusing on/including mixed populations of dementia and non-dementia (eg, cancer), when findings are not reported by disease group.

- Studies focusing on intellectual disabilities or Down syndrome 'AND' dementia; neurodegenerative diseases that are not dementia (such as Parkinson's disease and Huntington's disease) 'OR' particularly occur in early adulthood (eg, adult neuronal ceroid lipofuscinoses)

\section{Subject/intervention:}

- If the paper is explicitly referring to 'terminal care', or 'palliative care during the final week/month of life'. care provided, not the degree of training and/or experience of those providing care. Palliative care can be provided by any type of healthcare provider (GP, geriatrician, pharmacist, team, etc) and include both generalist and specialist palliative care (providers).

- Interventions, services delivery modelst, programmes, approaches to care or support, specific activities, tools, questionnaires that align with the aims of palliative care as defined by the WHO or one of its domains physical, psychological, social, spiritual.

- Interventions aimed at advance care planning, ADsł and (shared) decisionmaking.

- Palliative sedation when aimed to improve quality of life.

- Early palliative care is defined as 'in the early stages of the disease trajectory of dementia', starting from diagnosis

- Interventions solely focusing on music or other
- Pharmacological, medical interventions, stand-alone (palliative) therapies modifying the disease to prolong life (eg, palliative chemotherapy); specific treatments focused on relieving symptoms (eg, palliative radiotherapy, acetylcholinesterase inhibitor treatment; a cholinesterase inhibitor); specific medication or medication dosing or (de)prescribing, (dis) continuation of medication (without reference to broader framework of palliative care); nutrition (eg, specifically focusing on foregoing or stopping food and fluid, artificial hydration and nutrition, spoon feeding).

- Post-death interventions§ focusing on grief. arts-based interventions; if not placed within the broader context of palliative care.

- Articles focusing solely on physician-assisted dying, medical-aid-in-dying, assisted-dying, continuous sedation until death, end-of-life decision statements (focused on euthanasia); if not placed within the broader context of advance care planning.

- Articles focusing on costs or financial burden.

- Articles focusing on place of death or transitions in care (between healthcare settings) at the endof-life, rather than 'provision' of end-of-life or palliative care.

No restrictions on the types of study design.
Literature reviews and research using any methodology or method (ie, data is collected and analysed), both qualitative and quantitative are included. 


\begin{tabular}{lll}
\hline Inclusion criteria & Exclusion criteria \\
\hline Setting & We will include studies taking place in & Studies focusing on hospice(s) in USA (and other \\
the following (combination of) settings: & countries with same regulation and definition of \\
community dwelling, patient's home, & hospice) are excluded because it only involves last 6 \\
& outpatient/private clinic, community & months of life. \\
pharmacy, day care centre, emergency & \\
department, hospital, hospital discharge & \\
towards home or nursing home, nursing & \\
& home, residential care home, long-term & \\
care, memory clinics. & \\
\hline
\end{tabular}

${ }^{*}$ Types of dementia can include impaired cognitive function, (mild/severe) cognitive impairment, Alzheimer's disease, Lewy bodies, frontotemporal dementia, rapidly progressive dementia's (including prion disease or Jacob-Creutzfeldt, FFI, etc).

†Service delivery models are defined as those providing an overarching health or social care framework or system with multiple components and interacting elements for the organisation of care for people with dementia nearing the end of life and/or their caregivers.

‡Papers regarding advance medical treatment decisions (such as physician orders for life-sustaining treatment) are included in the study, if considered part of advance care planning.

§Predeath interventions and coping based psychosocial interventions if those are provided before death of patient with dementia and in early stages of the disease and if the intervention is explicitly linked to palliative care.

$\mathrm{AD}$, advance directive; GP, general practitioner; NA, not applicable.

frontotemporal dementia, Lewy bodies, prion disease, dementia with Huntington's or young-onset dementia), if data are available. Analysis will be performed using NVivo (QSR) software. We will collate, summarise, and map the literature, informed by tabulation, to represent quantitative and qualitative data visually (including study characteristics) ${ }^{61}{ }^{62}$ Characteristics of included studies are reported in a separate table, describing author, year of publication, study location (country), research aim/ questions, study design, study population/setting and sample size (n), methods, key findings and quality score.

We aim to develop a theoretical model of how, why and for whom early palliative care is relevant, while identifying a list of facilitators and barriers (and relationships between those) to its implementation reported. We additionally aim to report on a timeline/diagram with elements of definitions/conceptualisations of palliative care early in the disease trajectory and how those changed across time (and studies).

\section{Patient and public involvement}

The development of the research questions and measures were based on literature and public health efforts demonstrating that timely initiation of palliative care for persons with dementia is a priority for patients and families, and that all too often these services are unavailable or low quality. Patients and/or the public were not involved in the design, conduct, or reporting plans of this research. We aim to disseminate results through an academic publication and conferences targeting dementia care and palliative care providers.

\section{DISCUSSION}

In this scoping review, we will identify research regarding palliative care across various settings of dementia care, in particular related to its early initiation in the disease trajectory. A scoping review is an ideal method for the type of research questions that we propose.$^{50}$ This is a timely study, given the anticipated increase in dementia cases across the world and the escalating associated global suffering. Research regarding palliative care has, to a large extent, focused on people with advanced dementia rather than people with less severe dementia-potentially dying from other conditions-and it is unknown how people in the earlier stages with the disease are navigating palliative care services.

We anticipate results will identify research gaps and lay out much-needed basic principles for defining and conceptualising early palliative care in dementia. It will include a theoretical model about how, for who and why palliative care can be relevant in early stages of dementia, what are facilitators and barriers, and provide insight into what is the current timeline of how this concept evolved over time and studies. Even if our scoping review cannot generate results that can guide clinicians in deciding whether palliative care in early phases might be useful, why and what it should entail, we do expect we will be able to provide insight into what is known, what are current scientific debates and what are the knowledge gaps; to ultimately bridge some of the existing controversies regarding palliative care early in the disease trajectory of people living with dementia (or to at least highlight what is currently lacking and in what areas we need further research). We do not aim to provide clarity in estimating prognosis or to develop strict criteria for when the person living with dementia should have access to palliative care.

Our scoping review protocol has some limitations. A first key issue is that in order to limit the scope of the search, we anticipate excluding literature regarding care delivery similar to the US hospice system and therefore studies looking at the last 6 months of life; even if the population does not necessarily have advanced dementia. Given however that many people die without reaching the stages of advanced dementia, ${ }^{28}$ we might have excluded a 
particular group of people who have dementia and are in hospices or receive palliative care but are not necessarily identified as having advanced dementia. Second, because there is lack of a clear definition of what 'early palliative care' entails, the aim of this paper is focused on palliative care 'early in the disease trajectory' of people living with dementia. Third, given we have made a trade-off between the specificity of using dementia as a search term versus cognitive impairment, which includes many other problems besides dementia, we decided not to include cognitive impairment or dementia types other than 'Alzheimer's' in our search strategy. Applying a specific search string (ruling out non-diagnosed dementia) might lead to missing some relevant articles. When we report on results, we will highlight whether these are applicable to dementia or MCI (or distinct types of dementia), given that it is not sure whether each cognitive impairment will progress in dementia, hence people with cognitive impairment might not be needing the same information on prognosis, anticipating symptoms, challenges, etc. Fourth, our search string is searching for general palliative care and might therefore not be sensitive to specific interventions that are considered to be part of palliative care (eg, we may miss papers focusing on advance care planning, as this was not explicitly included in our search). Any deviations from the scoping review protocol outlined here, shall be noted in our final paper, along with a rationale and a reflection on the potential effect on the results.

\section{ETHICS AND DISSEMINATION}

A scoping review of published review articles is a novel approach for examining the breadth of literature. It does not require ethics approval. Although this is our first step in establishing a foundation for a research agenda, we will be disseminating results through a publication and conferences targeting dementia care and palliative care providers.

\section{Start date}

5 November 2019. Updated search: 5 February 2021.

\section{Anticipated completion date \\ End of September 2021.}

\author{
Author affiliations \\ ${ }^{1}$ Atlantic Fellow for Equity in Brain Health, Global Brain Health Institute (GBHI), \\ University of California, San Francisco, California, USA \\ ${ }^{2}$ End-of-Life Care Research Group, Department of Family Medicine \& Chronic Care, \\ Vrije Universiteit Brussel (VUB), Brussel, Belgium \\ ${ }^{3}$ Department of Family Medicine and Chronic Care, Vrije Universiteit Brussel (VUB), \\ Brussels, Belgium \\ ${ }^{4}$ Division of Palliative Care and Geriatric Medicine and the Mongan Institute for \\ Aging and Serious Illness, Massachusetts General Hospital, Boston, Massachusetts, \\ USA \\ ${ }^{5}$ Department of Physiological Nursing, University of California San Francisco, San \\ Francisco, California, USA \\ ${ }^{6}$ Department of Primary and Community Care, Radboud Universiteit Nijmegen, \\ Nijmegen, Gelderland, Netherlands
}

${ }^{7}$ Department of Public Health and Primary Care, Leiden University Medical Center, Leiden, Zuid-Holland, Netherlands

${ }^{8}$ UCSF Library, University of California, San Francisco, California, USA

${ }^{9}$ Center for Palliative Care, Harvard Medical School, Boston, Massachusetts, USA

\section{Twitter Joni Gilissen @JoniGilissen}

Acknowledgements The authors want to thank the UCSF librarians for their support, and the language editor for English language editing.

Contributors JG conceived of and developed the protocol, which was reviewed by all authors. PT and JG conceived of the search strategy. PT executed the search. $\mathrm{JG}, \mathrm{LH}, \mathrm{JvdS}, \mathrm{LVdB}$ and CR contributed to the development of the inclusion and exclusion criteria. The final manuscript was drafted by $J G$ and approved by $L H$, JvdS, LVdB, PT and CR.

Funding JG is a Senior Atlantic Fellow at the Atlantic Fellows for Equity in Brain Health Programme (Atlantic Philanthropies), Global Brain Health Institute (GBHI), University of California San Francisco (no grant number) and Maurange Fund Laureate, King Baudouin Foundation, Belgium (no grant number).

Competing interests None declared.

Patient and public involvement Patients and/or the public were not involved in the design, or conduct, or reporting, or dissemination plans of this research.

Patient consent for publication Not required.

Provenance and peer review Not commissioned; externally peer reviewed.

Open access This is an open access article distributed in accordance with the Creative Commons Attribution Non Commercial (CC BY-NC 4.0) license, which permits others to distribute, remix, adapt, build upon this work non-commercially, and license their derivative works on different terms, provided the original work is properly cited, appropriate credit is given, any changes made indicated, and the use is non-commercial. See: http://creativecommons.org/licenses/by-nc/4.0/.

\section{ORCID iD}

Joni Gilissen http://orcid.org/0000-0002-5388-495X

\section{REFERENCES}

1 Mitchell SL, Teno JM, Kiely DK, et al. The clinical course of advanced dementia. N Engl J Med Overseas Ed 2009;361:1529-38.

2 Livingston G, Sommerlad A, Orgeta V, et al. Dementia prevention, intervention, and care. The Lancet 2017;390:2673-734.

3 Weuve J, Hebert LE, Scherr PA, et al. Deaths in the United States among persons with Alzheimer's disease (2010-2050). Alzheimers Dement J Alzheimers Assoc 2014;10:e40-6.

4 Sampson EL, Anderson JE, Candy B, et al. Empowering better Endof-Life dementia care (EMBED-Care): a mixed methods protocol to achieve integrated person-centred care across settings. Int $J$ Geriatr Psychiatry 2020:gps.5251.

5 Harrison KL, Hunt LJ, Ritchie CS, et al. Dying with dementia: underrecognized and Stigmatized. J Am Geriatr Soc 2019;67:1548-51.

6 Murray SA, Kendall M, Mitchell G, et al. Palliative care from diagnosis to death. BMJ 2017;356:j878.

7 World Health Organization. Strengthening of palliative care as a component of integrated treatment throughout the life course 2014.

8 van der Steen JT, Radbruch L, Hertogh CMPM, et al. White paper defining optimal palliative care in older people with dementia: a Delphi study and recommendations from the European association for palliative care. Palliat Med 2014;28:197-209.

9 World Health Organization. Definition of palliative care. World Health organization (who), 2002. Available: http://who.int/cancer/palliative/ definition/en/ [Accessed 25 Jun 2015].

10 Zahradnik EK, Grossman H. Palliative care as a primary therapeutic approach in advanced dementia: a narrative review. Clin Ther 2014;36:1512-7

11 De Schreye R, Smets T, Deliens L, et al. Appropriateness of end-of-life care in people dying with dementia: applying quality indicators on linked administrative databases. J Am Med Dir Assoc 2020;21:S1525861019308904.

12 Maetens A, Deliens L, Van den Block L, et al. Are we evolving toward greater and earlier use of palliative home care support? A trend analysis using population-level data from 2010 to 2015. J Pain Symptom Manage 2019;58:19-28. e10.

13 Penders YWH, Gilissen J, Moreels S, et al. Palliative care service use by older people: time trends from a mortality follow-back study between 2005 and 2014. Palliat Med 2018;32:466-75. 
14 Beernaert K, Cohen J, Deliens L, et al. Referral to palliative care in COPD and other chronic diseases: a population-based study. Respir Med 2013;107:1731-9.

15 Harris-Kojetin L, Sengupta M, Park-Lee E, et al. Long-Term care providers and services users in the United States: data from the national study of long-term care providers, 2013-2014. Vital Health Stat 3 2016:x-xii; 1-105. x-xii;1-105.

16 Alzheimer's Association. Alzheimer's disease facts and figures 2020:391-460.

17 Aminifard O. Physician assessment of CMS eligibility criteria for hospice (PACE-H)., 2016. Available: https://escholarship.org/uc/item/ 56z8z17f [Accessed 14 Jan 2020].

18 Nakanishi M, van der Steen JT. National dementia plans to address escalating global palliative care needs. Palliat Med 2020;34:966-967.

19 van Riet Paap J, Mariani E, Chattat R, et al. Identification of the palliative phase in people with dementia: a variety of opinions between healthcare professionals. BMC Palliat Care 2015;14:56.

20 van der Steen JT, Radbruch L, de Boer ME, et al. Achieving consensus and controversy around applicability of palliative care to dementia. Int Psychogeriatr 2016;28:133-45.

21 Zapponi S, Ascari MC, Feracaku E, et al. The palliative care in dementia context: health professionals point of view about advantages and resistances. Acta Biomed 2018;89:45-54.

22 Giannouli V, Ivanova D, Stoyanova S, et al. When is a person with dementia in need of palliative care? opinions of healthcare professionals and university students from three South East European countries. Psychiatr Danub 2019;31:465-72.

23 van der Steen JT, Lemos Dekker N, Gijsberts M-JHE, et al. Palliative care for people with dementia in the terminal phase: a mixedmethods qualitative study to inform service development. BMC Palliat Care 2017:16:28.

24 Oosterveld-Vlug MG, Pasman HRW, Ten Koppel M, et al. Physician visits and recognition of residents' terminal phase in long-term care facilities: findings from the PACE cross-sectional study in $6 \mathrm{EU}$ countries. J Am Med Dir Assoc 2019;20:696-702.

25 van der Steen JT, Heymans MW, Steyerberg EW, et al. The difficulty of predicting mortality in nursing home residents. Eur Geriatr Med 2011;2:79-81.

26 Gill TM, Gahbauer EA, Han L, et al. Trajectories of disability in the last year of life. N Engl J Med 2010;362:1173-80.

27 Palmer JA, Smith AM, Paasche-Orlow RS, et al. Research literature on the intersection of dementia, spirituality, and palliative care: a scoping review. J Pain Symptom Manage 2020;60:116-134.

28 van der Steen JT, Ribbe MW, Deliens L, et al. Retrospective and prospective data collection compared in the Dutch end of life in dementia (DEOLD) study. Alzheimer Dis Assoc Disord 2014;28:88-94.

29 Beernaert K, Deliens L, De Vleminck A, et al. Is there a need for early palliative care in patients with life-limiting illnesses? interview study with patients about experienced care needs from diagnosis onward. Am J Hosp Palliat Care 2016;33:489-97.

30 Harris D. Forget me not: palliative care for people with dementia. Postgrad Med J 2007;83:362-6.

31 Beernaert K, Deliens L, Pardon K, et al. What are physicians reasons for not referring people with life-limiting illnesses to specialist palliative care services? A nationwide survey. PLoS One 2015; $10: e 0137251$

32 Annear MJ, Toye C, Mclnerney F, et al. What should we know about dementia in the 21st century? A Delphi consensus study. BMC Geriatr 2015;15:5.

33 Rossor MN, Fox NC, Mummery CJ, et al. The diagnosis of youngonset dementia. Lancet Neurol 2010;9:793-806.

34 International Alzheimer's Disease, World Health Organization, Editors. dementia: a public health priority. Geneva 2012.

35 Hughes J, Lloyd-Williams M, Sachs GA. Supportive care for the person with dementia. United Kingdom: Oxford University Press, 2010.

36 Nishimura M, Kohno A, van der Steen JT, et al. Conceptualization of a good end-of-life experience with dementia in Japan: a qualitative study. Int Psychogeriatr 2020;32:255-65.

37 Ten Koppel M, Onwuteaka-Philipsen BD, Van den Block L, et al. Palliative care provision in long-term care facilities differs across Europe: results of a cross-sectional study in six European countries (PACE). Palliat Med 2019;33:1176-88.

38 Pivodic L, Pardon K, Van den Block L, et al. Palliative care service use in four European countries: a cross-national retrospective study via representative networks of general practitioners. PLoS One 2013;8:e84440.
39 Amador S, Sampson EL, Goodman C, et al. A systematic review and critical appraisal of quality indicators to assess optimal palliative care for older people with dementia. Palliat Med 2019;33:415-29.

40 Kochovska S, Garcia MV, Bunn F, et al. Components of palliative care interventions addressing the needs of people with dementia living in long-term care: a systematic review. Palliat Med 2020;34:454-92.

41 Smith C, Newbury G. Palliative care for community patients diagnosed with dementia: a systematic review. Br J Community Nurs 2019;24:570-5.

42 Mataqi M, Aslanpour Z. Factors influencing palliative care in advanced dementia: a systematic review. BMJ Support Palliat Care 2020;10:145-156.

43 Erel M, Marcus E-L, Dekeyser-Ganz F. Barriers to palliative care for advanced dementia: a scoping review. Ann Palliat Med 2017;6:365-79.

44 Miranda R, Bunn F, Lynch J, et al. Palliative care for people with dementia living at home: a systematic review of interventions. Palliat Med 2019;33:726-42.

45 Senderovich $\mathrm{H}$, Retnasothie S. A systematic review of the integration of palliative care in dementia management. Palliat Support Care 2019:1-12.

46 Bolt SR, van der Steen JT, Schols JMGA, et al. Nursing staff needs in providing palliative care for people with dementia at home or in long-term care facilities: a scoping review. Int J Nurs Stud 2019;96:143-52.

47 Broady TR, Saich F, Hinton T. Caring for a family member or Friend with dementia at the end of life: a scoping review and implications for palliative care practice. Palliat Med 2018;32:643-56.

48 Gamondi C, Larkin P, Payne S. Core competencies in palliative care: an EAPC white paper on palliative care education: Part 2. Eur $J$ Palliat Care 2013.

49 Levac D, Colquhoun H, O'Brien KK. Scoping studies: advancing the methodology. Implement Sci 2010;5:69.

50 O'Brien KK, Colquhoun H, Levac D, et al. Advancing scoping study methodology: a web-based survey and consultation of perceptions on terminology, definition and methodological steps. BMC Health Serv Res 2016;16:305.

51 Dixon-Woods M, Agarwal S, Jones D, et al. Synthesising qualitative and quantitative evidence: a review of possible methods. J Health Serv Res Policy 2005;10:45-53.

52 Tricco AC, Lillie E, Zarin W, et al. PRISMA extension for scoping reviews (PRISMA-ScR): checklist and explanation. Ann Intern Med 2018;169:467-73.

53 Sladek R, Tieman J, Fazekas BS, et al. Development of a subject search filter to find information relevant to palliative care in the general medical literature. J Med Libr Assoc 2006;8.

54 Sladek RM, Tieman J, Currow DC. Improving search filter development: a study of palliative care literature. BMC Med Inform Decis Mak 2007;7:18.

55 Mitchell JC, Dick MB, Wood AE, et al. The utility of the dementia severity rating scale in differentiating mild cognitive impairment and Alzheimer disease from controls. Alzheimer Dis Assoc Disord 2015;29:222-8

56 Rikkert MGMO, Tona KD, Janssen L, et al. Validity, reliability, and feasibility of clinical staging scales in dementia: a systematic review. Am J Alzheimers Dis Other Demen 2011;26:357-65.

57 Creavin ST, Wisniewski S, Noel-Storr AH, et al. Mini-Mental state examination (MMSE) for the detection of dementia in clinically unevaluated people aged 65 and over in community and primary care populations. Cochrane Database Syst Rev 2016:CD011145.

58 Moher D, Liberati A, Tetzlaff J, et al. Preferred reporting items for systematic reviews and meta-analyses: the PRISMA statement. Ann Intern Med 2009:151:264-9.

59 Mays N, Pope C, Popay J. Systematically reviewing qualitative and quantitative evidence to inform management and policy-making in the health field. J Health Serv Res Policy 2005;10 Suppl 1:6-20.

60 Braun V, Clarke V. Using thematic analysis in psychology. Qual Res Psychol 2006;3:77-101.

61 Popay J, Roberts H, Sowden A, et al. Guidance on the conduct of narrative synthesis in systematic reviews. Prod ESRC Methods Programme Version 2006; 1

62 Rodgers M, Sowden A, Petticrew M, et al. Testing methodological guidance on the conduct of narrative synthesis in systematic reviews: effectiveness of interventions to promote smoke alarm ownership and function. Evaluation 2009;15:49-73. 